\title{
HST Resolves Stars in a Tiny Body Falling on the Dwarf Galaxy DDO 68
}

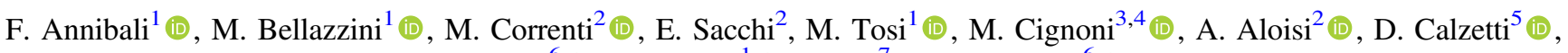 \\ L. Ciotti ${ }^{6}$ (D) F. Cusano ${ }^{1}$ (D) , J. Lee ${ }^{7}$, and C. Nipoti ${ }^{6}$ (D) \\ ${ }^{1}$ INAF-Astrophysics and Space Science Observatory, Via Piero Gobetti, 93/3, I-40129—Bologna, Italy; francesca.annibali@inaf.it \\ ${ }^{2}$ Space Telescope Science Institute, 3700 San Martin Drive, Baltimore, MD 21218, USA \\ ${ }^{3}$ Dipartimento di Fisica, Università di Pisa, Largo Bruno Pontecorvo, 3, I-56127 Pisa, Italy \\ ${ }^{4}$ INFN, Sezione di Pisa, Largo Bruno Pontecorvo, 3, I-56127 Pisa, Italy \\ ${ }^{5}$ Department of Astronomy, University of Massachusetts-Amherst, 710 N Pleasant Street, Amherst, MA 01003-9305, USA \\ ${ }^{6}$ Dipartimento di Fisica e Astronomia, Università di Bologna, Via Gobetti 93/2, I-40129 Bologna, Italy \\ ${ }^{7}$ Caltech-IPAC, 1200 E California Blvd, Pasadena, CA 91125, USA \\ Received 2019 April 2; revised 2019 July 9; accepted 2019 July 19; published 2019 September 17
}

\begin{abstract}
We present new Hubble Space Telescope imaging of a stream-like system associated with the dwarf galaxy DDO 68, located in the Lynx-Cancer void at a distance of $D \sim 12.65 \mathrm{Mpc}$ from us. The stream, previously identified in deep Large Binocular Telescope images as a diffuse low surface brightness structure, is resolved into individual stars in the F606W (broad $V$ ) and F814W $(\sim I)$ images acquired with the Wide Field Camera 3. The resulting $V, I$ color-magnitude diagram $(\mathrm{CMD})$ of the resolved stars is dominated by old (age $\gtrsim 1-2 \mathrm{Gyr}$ ) red giant branch (RGB) stars. From the observed RGB tip, we conclude that the stream is at the same distance as DDO 68, confirming the physical association with it. A synthetic CMD analysis indicates that the large majority of the star formation activity in the stream occurred at epochs earlier than $\sim 1 \mathrm{Gyr}$ ago, and that the star formation at epochs more recent than $\sim 500 \mathrm{Myr}$ ago is compatible with zero. The total stellar mass of the stream is $\sim 10^{6} \mathrm{M}_{\odot}$, about $1 / 100$ of that of DDO 68. This is a striking example of hierarchical merging in action at the dwarf galaxy scales.
\end{abstract}

Key words: galaxies: dwarf - galaxies: interactions - galaxies: irregular - galaxies: star formation - galaxies: stellar content

\section{Introduction}

The dwarf galaxy DDO 68, with a stellar mass of $\sim 10^{8} M_{\odot}$ (Sacchi et al. 2016) and a dynamical mass of $M_{\mathrm{dyn}} \gtrsim 5.2 \times$ $10^{9} M_{\odot}$ (Cannon et al. 2014), is a rare observed example of a merger occurring at the scale of dwarf galaxies. Located at a distance of $\sim 12.65 \mathrm{Mpc}$ from us (Sacchi et al. 2016), it resides in a very empty region of space, in the Lynx-Cancer void (Pustilnik \& Tepliakova 2011); nevertheless, it has been suggested to be in the process of accreting (or interacting with) one or more smaller companions. Ekta et al. (2008) hypothesized a late-stage merger between two gas-rich progenitors to explain the observed distortions in the HI. Using Hubble Space Telescope (HST) data, Tikhonov et al. (2014) proposed that DDO 68 is in fact composed of two distinct systems, a main component, DDO $68 \mathrm{~A}$, and a disrupted satellite (dubbed DDO 68 B) being currently accreted by DDO $68 \mathrm{~A}$ and producing the "cometary tail." Cannon et al. (2014) detected a more remote, low-mass $\left(M_{\mathrm{H}} \sim 3 \times 10^{7} M_{\odot}\right)$ companion, located at a projected distance of $\sim 40 \mathrm{kpc}$ from DDO 68 and connected to the galaxy through a bridge of low surface brightness $\mathrm{H}$ I gas $\left(N_{\mathrm{H} \mathrm{I}} \sim 2 \times 10^{18} \mathrm{~cm}^{-2}\right)$. Indeed, the occurrence of mergers and interaction events could help explain some of the peculiarities observed in DDO 68, such as its very distorted morphology, characterized by the presence of a "cometary tail," the unusual distribution of its $\mathrm{H}$ II regions, mostly located in the head and tail of the "comet" and almost absent in the innermost galaxy regions (Moiseev 2014; Pustilnik et al. 2017), and its very low metallicity ( $2 \%$ solar, e.g., Pustilnik et al. 2005; Izotov \& Thuan 2009; Annibali et al. 2019, which is one of the lowest metallicities ever measured in a star-forming galaxy), much too low for its mass. For instance, Lee et al. (2004) showed that the inclusion of interacting galaxies can increase the scatter in the luminosity-metallicity relation and may force the observed correlation toward lower metallicities and/or larger luminosities/ masses.

In Sacchi et al. (2016), we derived the star formation history (SFH) of DDO 68 using HST Advanced Camera for Surveys (ACS) data in F606W ( broad $V$ ) and F814W $(\sim I)$. We found that DDO 68, despite its extremely low metallicity, hosts a conspicuous population of red giant branch (RGB) stars, implying that the galaxy has been forming stars since at least 1-2 Gyr ago, and possibly since epochs as early as a Hubble time. The galaxy star formation activity has been fairly continuous over all the look-back time, with an enhancement by factor of $\gtrsim 2$ between 10 and $100 \mathrm{Myr}$ ago (at a rate of $\sim 4 \times 10^{-2} \widetilde{M_{\odot}} \mathrm{yr}^{-1} \mathrm{kpc}^{-2}$ ), and a lower activity at current epochs. Interestingly, when studying the spatial distribution of stars of different ages, we recognized a peculiar concentration of RGB stars toward an edge of the ACS chip, as also identified by Tikhonov et al. (2014). We noticed that the magnitudes of these stars were compatible with DDO 68's distance but, due to the poor statistics, we could not draw firm conclusions.

With the aim of getting insights into the origin of these RGB stars, and into DDO 68's merging history more in general, we exploited the powerful combination of a large field of view $\left(\sim 23 \times 23 \operatorname{arcmin}^{2}\right)$ and high photometric depth provided by the Large Binocular Cameras (LBC) on the Large Binocular Telescope (LBT), and acquired new deep $g$ - and $r$ - imaging of DDO 68. The LBT data showed that the peculiar concentration of RGB stars detected in the ACS images are in fact part of a more extended low surface brightness $\left(\mu_{\mathrm{r}} \sim 28.7 \mathrm{mag} \operatorname{arcsec}^{-2}\right)$ stream-like system connected to DDO 68, which we dubbed S1 (Annibali et al. 2016). Figure 1 displays a portion of our LBT image with the stream S1 and an identified low surface brightness arc indicated. S1 has a projected size of $\sim 20 \times 80 \operatorname{arcsec}^{2}$ in the LBT images, a total 


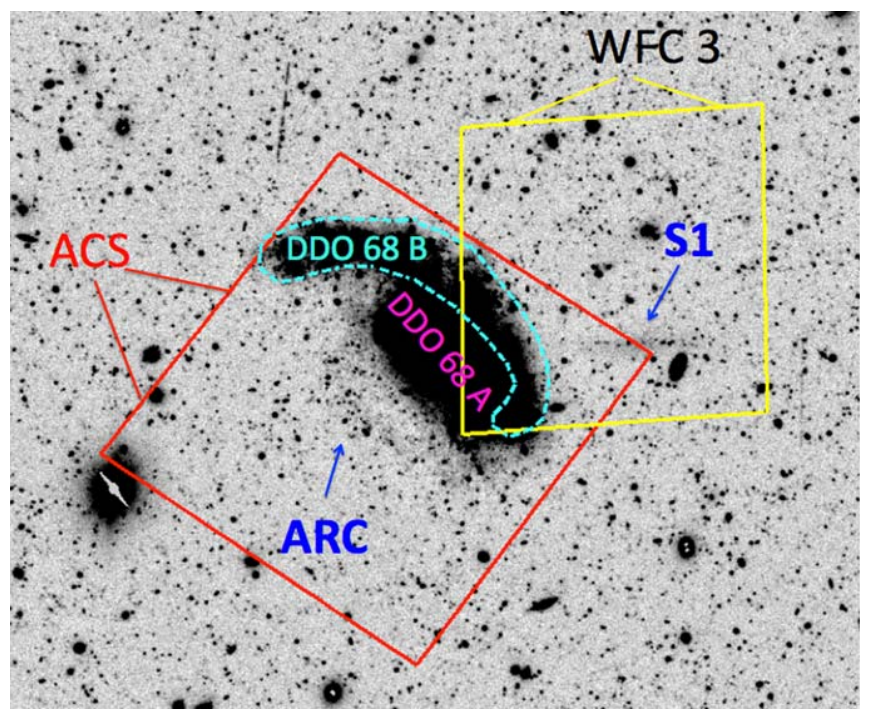

Figure 1. $500 \times 400 \operatorname{arcsec}^{2}$ portion of our LBT LBC image of DDO 68 showing the arc and the stream S1 identified by Annibali et al. (2016). The dashed cyan contour indicates the approximate division of DDO 68 into the A and B subsystems, as proposed by Tikhonov et al. (2014). Overplotted on the LBT image are the footprints of our previously acquired ACS data (Sacchi et al. 2016) and of the new WFC3 data.

integrated magnitude of $m_{r}=21.6 \pm 0.4 \mathrm{mag}$, and an average color of $g-r=0.56 \pm 0.11$, which translate, under the assumption of the same DDO 68's distance of $\sim 12.65 \mathrm{Mpc}$, into a physical size of $\sim 1.2 \times 5 \mathrm{kpc}^{2}$ and a stellar mass in the range (1.5-6) $\times 10^{5} M_{\odot}$. In Annibali et al. (2016) we showed through $N$-body simulations that while DDO 68's "cometary tail" can be reproduced by the accretion of a ten times less massive body, S1 requires the accretion of a third body with a mass of about one hundredth that of DDO 68.

In this paper we present new HST Wide Field Camera 3 (WFC3)/Ultraviolet-Visible channel (UVIS) data acquired during Cycle 24 with the main purposes of resolving the entire S1 system into individual stars, improving the statistics with respect to our previous ACS data, and deriving a conclusive distance for S1 via the RGB tip method. Probing the physical association of S1 with DDO 68 through its distance is a fundamental step to confirm a rare observed case of multiple accretions onto an isolated dwarf galaxy as small as DDO 68.

Indeed, $N$-body simulations in a $\Lambda$ cold dark matter ( $\Lambda \mathrm{CDM}$ ) cosmology predict that structures are assembled through a continuous hierarchical merging process, and a consequence is that present-day galaxies, both giants and dwarfs, are expected to be surrounded and orbited by a large number of smaller systems or satellites (e.g., Diemand et al. 2008). However, while there is ample observational evidence for this to occur around giant galaxies (e.g., in the Milky Way (Belokurov et al. 2006), in Andromeda (Ibata et al. 2001; McConnachie et al. 2009), in several Local Volume spirals (Martínez-Delgado et al. 2010) and also giant ellipticals such as Centaurus A (CenA; Crnojević et al. 2016)), little observational evidence of accretion events into LMC-size galaxies has been reported in the literature so far. Among these rare examples are the LMC itself, with several convincing satellites (Koposov et al. 2018), and the magellanic irregular galaxy NGC 4449, with evidence for a stellar stream (Martínez-Delgado et al. 2012; Rich et al. 2012) and for another disrupted dwarf companion (Annibali et al. 2012). However, accretion phenomena onto galaxies less massive than $\sim 10^{9} M_{\odot}$ in stars have remained elusive so far (Amorisco et al. 2014; Kacharov et al. 2017; Cicuéndez \& Battaglia 2018), although panoramic imaging surveys have revealed possible candidate satellites of dwarf galaxies (the Antlia B dwarf, a likely satellite of the dwarf galaxy NGC 3109 (Sand et al. 2015), and Dw2, likely associated to the ultradiffuse dwarf galaxy Dw1 in the CenA group (Crnojević et al. 2019)) and very compact groups composed of only dwarfs (Stierwalt et al. 2017). Therefore the striking case of DDO 68 is a rare observed example of hierarchical merging in action at the dwarf galaxy scales predicted by the $\Lambda$ CDM scenario.

The paper is structured as follows. Observations and data reduction are described in Section 2. The stream's colormagnitude diagram (CMD) is presented in Section 3, while we investigate the spatial distribution of the different stellar populations in Section 4. In Section 5 we provide evidence that $\mathrm{S} 1$ is located at the same distance of DDO 68 from us. In Section 6 we derive S1's SFH through the method of the synthetic CMDs. Finally, we present our discussion and conclusions in Section 7.

\section{Observations and Data Reduction}

The observations were performed in 2017 December with the WFC3/UVIS channel in the F606W (broad $V$ ) and F814W (I) broadband filters (GO program 14716, PI: Annibali). We preferred the WFC3 to the ACS because of the ageing of ACS and the more severe impact of charge transfer efficiency (CTE) effects compared to WFC3, and the F606W filter to the F555W filter because of the higher sensitivity of the former. This is the same filter setup adopted in our previous ACS program centered on DDO 68 (GO program 11578; PI: Aloisi), allowing for a direct comparison between the photometry.

The WFC3/UVIS camera was positioned with a specific orientation, with the gap between the two detectors rotated by $\sim 55^{\circ} \mathrm{NE}$, in order to place the whole S1 system within chip 2, thus avoiding the gap (see Figure 2). A portion of the WFC3 field of view intentionally overlaps with our previous ACS observations of DDO 68. We observed S1 for total exposure times of $\sim 13,000 \mathrm{~s}$ in $\mathrm{F} 606 \mathrm{~W}$ and $\sim 13,000 \mathrm{~s}$ in $\mathrm{F} 814 \mathrm{~W}$, implying a total of $10 \mathrm{HST}$ orbits. The exposure times were determined assuming S1 was at the same distance as DDO 68 $\left(\sim 12.65 \mathrm{Mpc}\right.$ or $(m-M)_{0} \sim 30.51$; Sacchi et al. 2016) and requiring to reach down to 1 mag below the tip of the RGB (i.e., down to $I \sim 27.4, V \sim 28.7$ ) with a signal-to-noise ratio of $\sim 5$. For each filter, the five HST orbits were split into two separate visits lasting two and three orbits, respectively. Each visit was organized into eight sub-exposures, executed with a primary 2-point, $\sim 2$ ". 4 separation dither pattern to fill the gap between the WFC3 chips, combined with a secondary 4-point half + integer pixel dither pattern to improve the point-spread function (PSF) sampling, and get rid of cosmic rays and hot/ bad pixels. Within the longer 3-orbit visit, we added two short $\sim 300 \mathrm{~s}$ exposures, executed with a 2-point spacing dither pattern ( 4 ". 8 separation), in order to get photometry of bright objects saturated in the long exposures.

We retrieved from the $H S T$ archive the $*$.flc science images, which are the bias-corrected, dark-subtracted, flat-fielded, and CTE corrected data products from the CALWF3 pipeline (Ryan et al. 2016). The individual $*$.flc images were then combined together into a single stacked, distortion-corrected reference image (i.e., the *.drc image) using the Drizzlepac software (Gonzaga et al. 2012). More specifically, we first aligned all the 

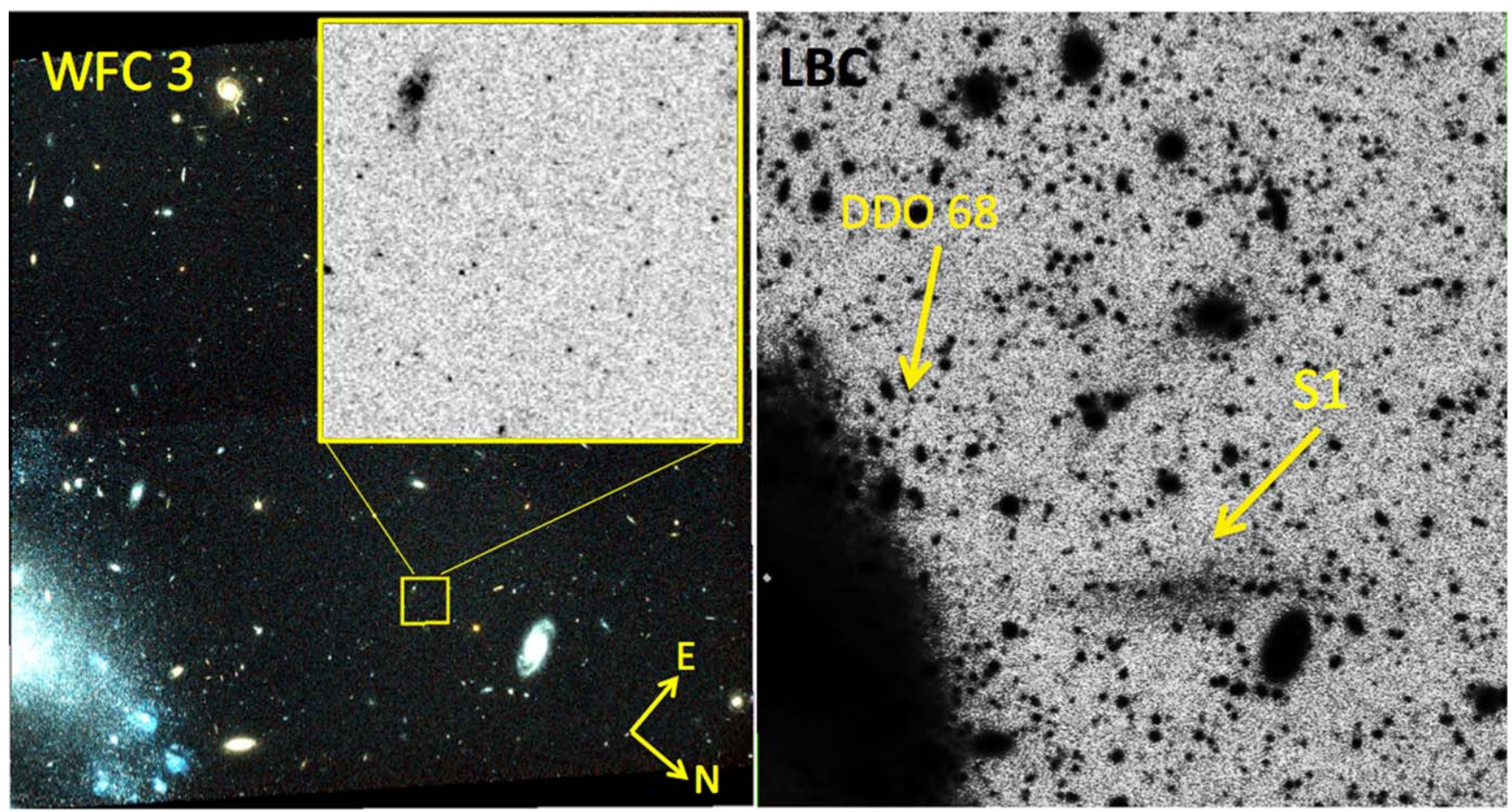

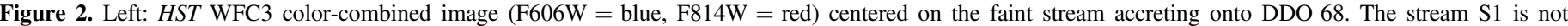

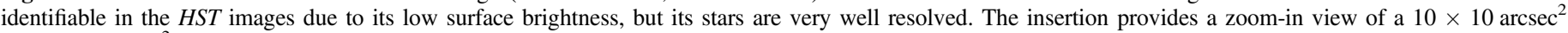

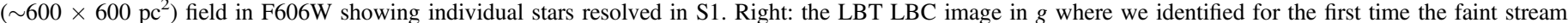
(Annibali et al. 2016).

images in the same filter using the software TweakReg, with the final transformations providing alignment of individual images to better than 0.02 pixels. Then, using the software AstroDrizzle, bad pixels and cosmic rays were flagged and rejected from the input images, and the input undistorted and aligned frames were combined together into a final stacked image. The final stacked images were generated at the native resolution of the WFC3/UVIS camera (i.e., 0."04/pixel ${ }^{-1}$ ).

To perform stellar photometry, we used the latest version of Dolphot (Dolphin 2000, and numerous subsequent updates). This software includes pre-processing and photometry modules customized to each HST camera as well as precomputed PSFs tailored to each filter. PSF fitting photometry was performed on the individual ${ }^{*}$ flc images using the combined drizzled ${ }^{*}$ drc image as a reference frame. In order to achieve an optimal image alignment and source detection, the Dolphot parameters were set using a hybrid combination between the values recommended by the Dolphot manual and those adopted in Williams et al. (2014).

The final output photometric catalog contains position and photometry of individual stars in F606W, F814W Vegamag, as well as several diagnostic parameters that can be used to exclude remaining artifacts and spurious detections. From the total catalog, we selected objects with a Dolphot "object type" flag of 1, corresponding to "good stars" that are well fitted by the PSF. However, background contaminants and spurious detections are likely to be still present in the catalog, and therefore we applied further cuts in the sharpness versus magnitude and error versus magnitude planes, as shown in Figure 3. Similar selections have proven to be very effective in removing a major fraction of contaminants from the photometric catalogs in our previous studies of dwarf galaxies (e.g., Sacchi et al. 2016). We notice in particular that the error versus magnitude distributions in both F606W and F814W consist of two separate "sequences," with the "upper" one being only populated by spurious detection at the edges of the WFC3 chips.

Confusion with background galaxies is a potential issue given the relatively low number of stars that we expect to resolve in $\mathrm{S} 1$; for this reason, we eventually performed a visual inspection of the individual sources on the WFC3 colorcombined $V$, I image to remove obvious background galaxies that had survived to our selection cuts. We caution, however, that very compact background galaxies, indistinguishable from individual stars, will still be present in the final catalog. Our final cleaned photometric catalog consists of $\sim 10,800$ objects.

A portion of the WFC3 field of view overlaps part of the ACS images presented by Sacchi et al. (2016). For a consistency check between the ACS and WFC3 photometry, we cross-correlated our catalog with that of Sacchi et al. (2016) and directly compared the F606W and F814W magnitudes for the stars in common between the two catalogs. Although the ACS and WFC3 photometries agree quite well within the errors, we notice the presence of a small systematic 0.03-0.04 offset in both filters between the two data sets, with the Sacchi et al. magnitudes being fainter than ours. We notice that this discrepancy can not be ascribed to photometric calibration differences between ACS and WFC3 (Deustua \& Mack 2018), and it is likely due to uncertainties in the applied aperture corrections.

\subsection{Artificial Star Tests}

Artificial star tests were performed on the WFC3 images in order to evaluate the incompleteness and the photometric errors as a function of magnitude. Indeed, the errors produced by photometric packages are typically underestimated (e.g., 

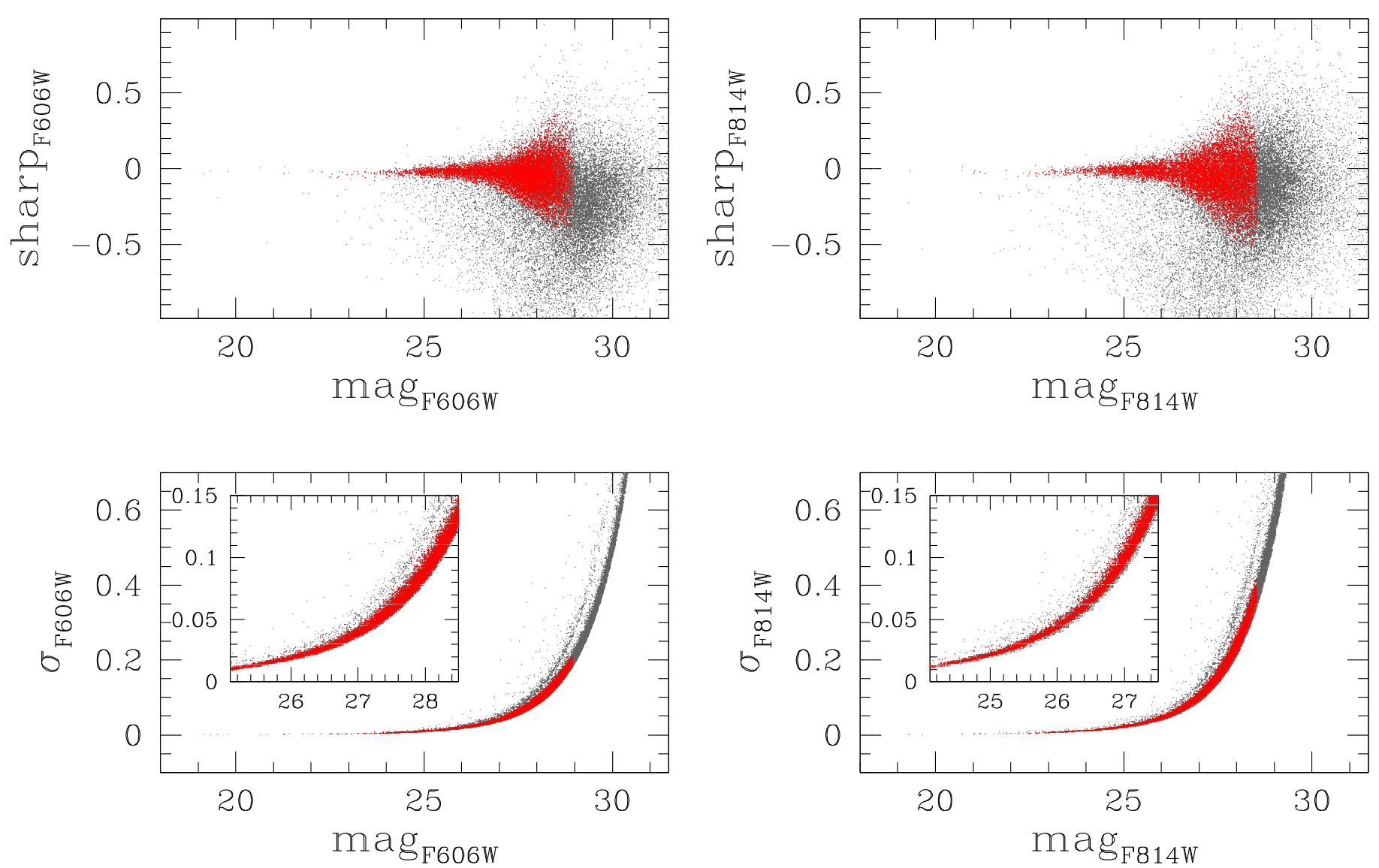

Figure 3. Dolphot sharpness parameter vs. magnitude (top panels) and magnitude error vs. magnitude (bottom panels) in both F606W and F814W. Gray dots represent all sources selected adopting a Dolphot object type flag of 1, as described in Section 2, while red dots denote our adopted selections in the four planes. The two insertions in the bottom panels provide a zoom-in view of our error selection excluding sources in the "upper" sequences; these objects correspond to spurious detections on the WFC 3 chip edges.

Williams et al. 2014) and do not provide a realistic evaluation of the photometric uncertainties. The adopted method, described in detail in previous papers (e.g., Cignoni et al. 2016; Sacchi et al. 2016), consists of adding artificial stars to the images and then in reducing the frames following the same procedure as for the real data. Artificial stars were injected into the images following a uniform distribution; furthermore, they were added one at a time to not artificially alter the crowding on the images. Color and magnitude intervals of the artificial stars were chosen to cover the observed CMD range and reaching down to $\sim 3$ mag fainter than the observed magnitude limits. Each artificial star of given magnitude and color was then injected simultaneously into the F606W and F814W frames at one position in order to reproduce a realistic situation and to account for the correlation between the completeness in the two bands. From the artificial star output catalog, a star was considered "recovered" if it had a measured magnitude within $0.75 \mathrm{mag}$ from its input value in both filters, and if it satisfied all the selection cuts that were applied to the real photometry (see Section 2, Figure 3). Our tests provide a 50\% completeness at F606W $\sim 28$ and F814W $\sim 27.5$ in the crowded main body of DDO 68, and at F606W $~ 28.5$ and F814W $\sim 28$ in the less crowded outer field. From the output minus input magnitude distributions, we estimate $1 \sigma$ photometric errors of $\sim 0.06$ and $\sim 0.13$ at F606W $=27$ and 28 , and of $\sim 0.16$ and $\sim 0.32$ at $\mathrm{F} 814 \mathrm{~W}=27$ and 28 .

\section{Color-Magnitude Diagram}

The total cleaned F814W versus F606W-F814W CMD for the sources measured in the whole WFC3 field is shown in the top panel of Figure 4, while in the middle and bottom panels we compare the observed CMD with the PARSEC+COLIBRI stellar isochrones (Bressan et al. 2012; Marigo et al. 2017) for two different metallicities: one of $Z=0.0004(\sim 1 / 40$ solar $)$, consistent with the measured DDO 68's H II region oxygen abundance, and the other of $Z=0.004$, which is more compatible with the metallicity expected from the galaxy total luminosity. The displayed CMD was not corrected for reddening, while the isochrones were shifted assuming a distance modulus of $(m-M)_{0}=30.54$ (see Section 5) and a foreground reddening of $E(B-V)=0.016$ (Schlafly \& Finkbeiner 2011); internal dust extinction within $S 1$ is expected to be negligible given the modest $\mathrm{HI}$ column densities at the position of S1 (Cannon et al. 2014) and the extremely low metallicity of DDO 68.

The CMD exhibits all the evolutionary features typically observed in late-type dwarf galaxies: a well defined blue plume at F606W-F814W $\sim-0.05$ and F814W $\lesssim 26.5$, populated by massive, young (age $\lesssim 10 \mathrm{Myr}$ ) main-sequence stars and hot post-main-sequence stars (age $\lesssim 50 \mathrm{Myr}$ ); a red plume at F606W-F814W $\sim 1$ and $\mathrm{F} 814 \mathrm{~W} \lesssim 26.5$, containing red supergiants and asymptotic giant branch (AGB) stars with ages from $\sim 10$ Myr up to a few Gyr; the red horizontal feature 


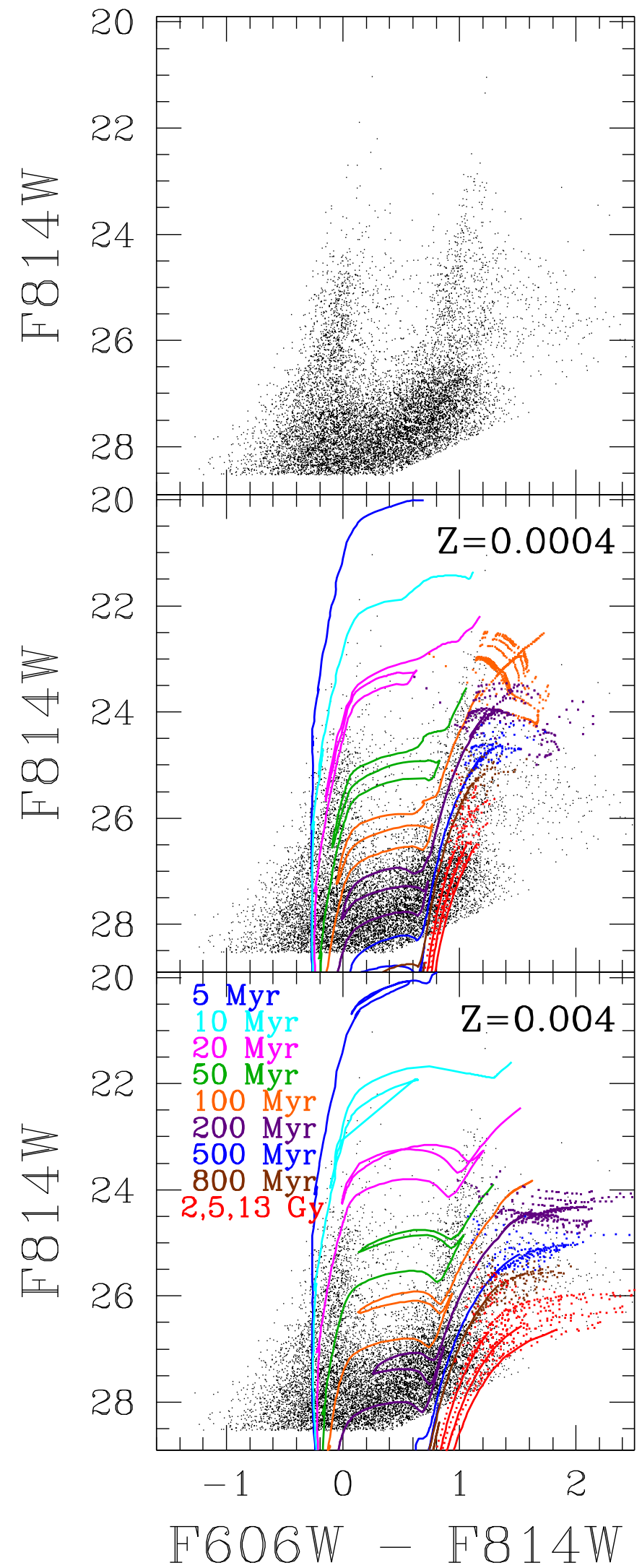

Figure 4. $I, V-I$ color-magnitude diagram for sources in our final cleaned photometric catalog (see Section 2 for details). Superimposed are the PARSEC stellar isochrones (Bressan et al. 2012; Marigo et al. 2017) for metallicities $Z=0.0004$ (middle panel) and $Z=0.004$ (bottom panel), and for different ages as indicated in the legend. The isochrones have been displayed assuming a distance modulus of $(m-M)_{0}=30.54$ (see Section 5) and a foreground reddening of $E(B-V)=0.016$. at $\mathrm{F} 814 \mathrm{~W} \sim 25.5$ and $1 \lesssim \mathrm{F} 606 \mathrm{~W}-\mathrm{F} 814 \mathrm{~W} \lesssim 2.5$ due to carbon AGB stars in the thermal pulses phase (hereafter, TPAGB), with ages from a few hundred Myr up to a couple Gyr; intermediate color objects at $0 \lesssim$ F606W-F814W $\lesssim 0.8$, $\mathrm{F} 814 \mathrm{~W}>26.5$, which are intermediate-mass, core heliumburning stars with ages between $\sim 100$ and $\sim 300$ Myr (older stars in this evolutionary phase fall below our detection limit); finally, a concentration of objects at F814W $>26.5$ and $0.6 \lesssim \mathrm{F} 606 \mathrm{~W}-\mathrm{F} 814 \mathrm{~W} \lesssim 1.2$, which is due to RGB stars, and implies ages older than $1-2 \mathrm{Gyr}$ and potentially as old as the age of the universe. The same evolutionary features were observed by Sacchi et al. (2016) for their CMD of the entire DDO 68 galaxy obtained from ACS data.

Figure 4 shows that the overall appearance of the observed CMD is qualitatively well reproduced by the $Z=0.0004$ models, in agreement with the previous analysis of Sacchi et al. (2016). In particular, the F606W-F814W color of the RGB is compatible with isochrones in the $2-13 \mathrm{Gyr}$ age range, while models as metal-rich as $Z=0.004$ provide a poor match to this evolutionary phase unless ages of $\lesssim 2 \mathrm{Gyr}$ are assumed. On the other hand, the F606W-F814W color of younger stars (ages $\lesssim 100 \mathrm{Myr}$ ) in the blue and red plumes is less sensitive to the effect of metallicity, and strong claims concerning their metal content are not possible. Finally, we notice that the $Z=0.0004$ models are not able to reproduce the observed red horizontal feature due to TP-AGB stars, which seems to better agree with models of higher metallicity. Indeed, DDO 68 is a strong outlier in the mass-metallicity relation, and its extremely low $\mathrm{H}$ II region oxygen abundance has been suggested to be due to the accretion of metal-poor gas; admittedly, we can not definitely exclude from the observed CMD the presence of a relatively young (age $\lesssim 1-2 \mathrm{Gyr}$ ) stellar population with metallicity several times higher than that observed in its H II regions (see also Tikhonov et al. 2014).

\section{Resolved Star Maps}

We investigated how stars in the total cleaned photometric catalog are distributed over the WFC3 field of view. Figure 5 shows spatial distributions for the totality of stars and for groups of stars belonging to different age intervals, as selected from the CMD. Guided by the stellar isochrones displayed in Figure 4, we identified four regions in the CMD roughly corresponding to four different age intervals (see top left panel of Figure 5): blue plume stars with ages $\lesssim 50 \mathrm{Myr}$; red plume stars, in the range $\sim 10 \mathrm{Myr}-\sim 200 \mathrm{Myr}$; AGB stars with ages in the range $\sim 200$ Myr- 2 Gyr; RGB stars, with ages older than 1-2 Gyr, and potentially as old as $\sim 13$ Gyr. The spatial distribution of the totality of stars in the top central panel of Figure 5 undoubtedly shows that the faint stream S1 identified in our previously acquired LBT images is resolved into individual stars in the WFC3 data. No obvious substructure other than $\mathrm{S} 1$ is identified in the resolved star maps. While the DDO 68 portion sampled by our WFC3 data contains stars of all ages, in agreement with the findings of Sacchi et al. (2016), S1 does not host young stars, and it is only populated by intermediate-age and old stars.

DDO 68 is also one of the 50 star-forming galaxies targeted by the LEGUS HST Treasury program (ID 13364, PI: Daniela Calzetti; see e.g., Calzetti et al. 2015); hence, WFC3/UVIS photometry is available in the F275W, F336W, and F438W bands in a field of view that partially also covers the stream. No bona fide star can be identified in the stream in any of these short-wavelength images, in agreement with the lack of a 

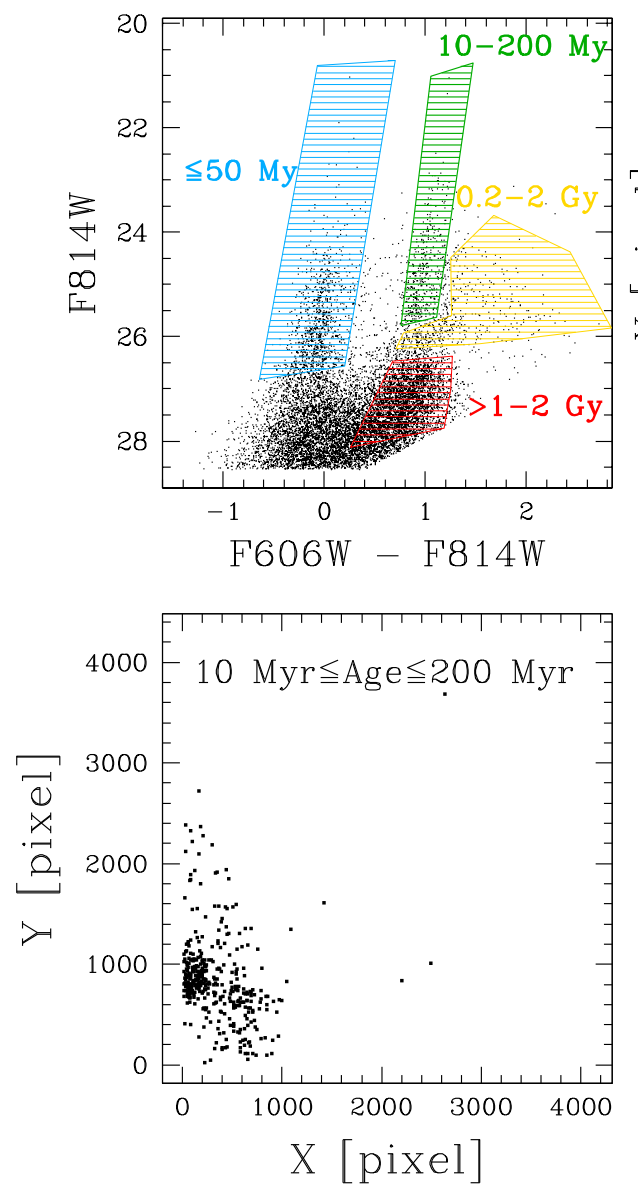
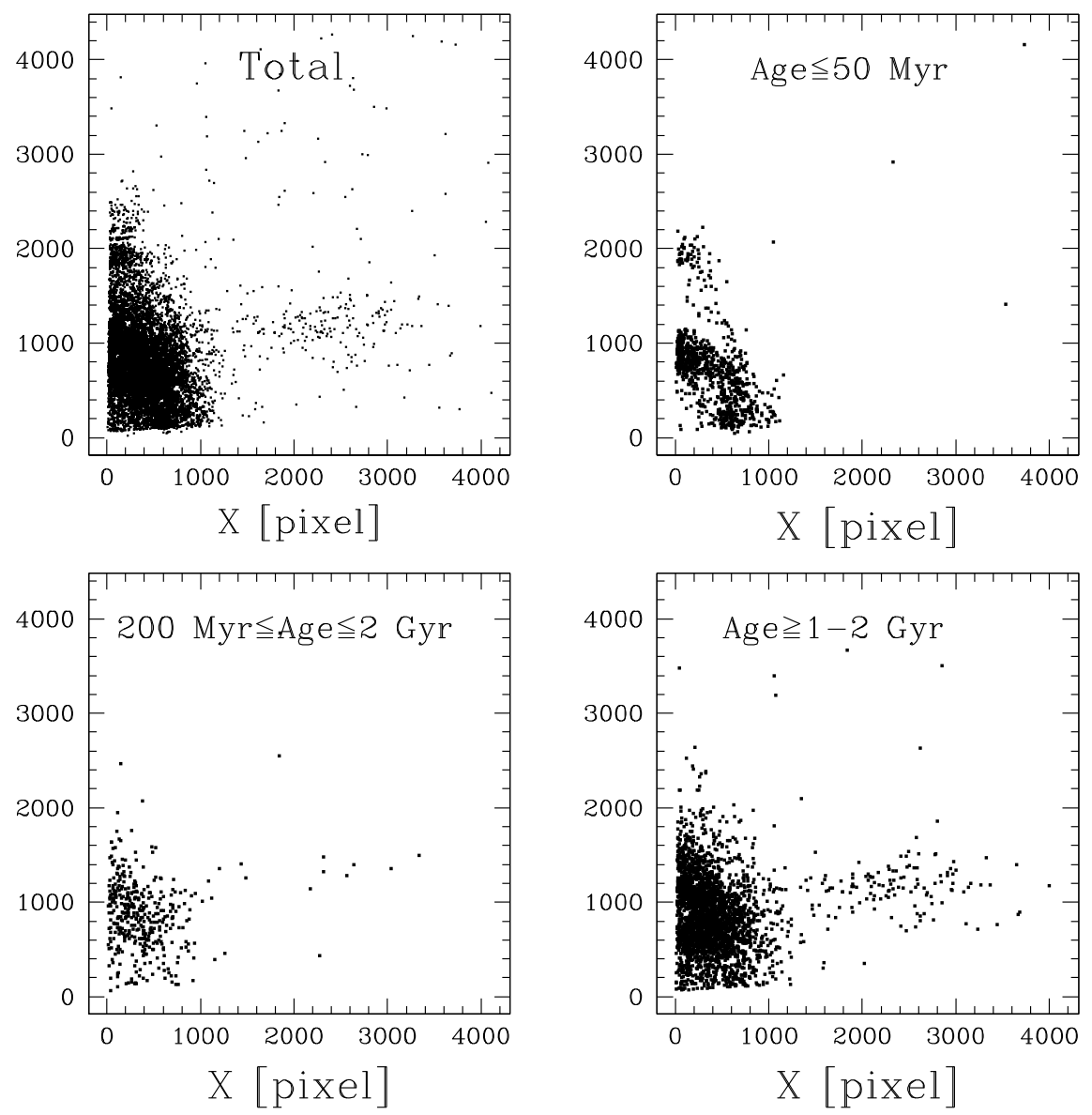

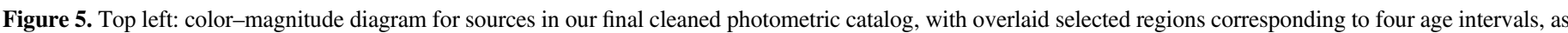

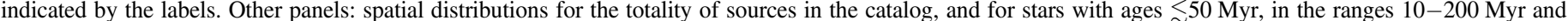

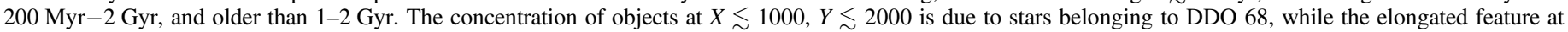
$1400 \lesssim X \lesssim 3400,800 \lesssim Y \lesssim 1600$ is the stream-like $\mathrm{S} 1$ system.

young population in the F606W, F814W CMD. A more indepth investigation of the S1's SFH will be performed in Section 6 through synthetic CMDs.

\subsection{Structural Properties of S1}

Stars belonging to different age intervals, as selected from the CMD in Figure 5, are overplotted on the LBT LBC image in Figure 6 for a direct comparison. The resolved stars perfectly overlap with the unresolved low surface brightness feature identified in the LBT data. We can thus use the spatial distribution of AGB and RGB stars to infer some structural parameters of S1: the resolved star map is best fitted by ellipses with major axis oriented along a position angle of $\mathrm{PA}=36_{-18}^{+10} \mathrm{deg}(\mathrm{N}$ to $\mathrm{E}$ ), and minor over major axis ratio of $b /$ $a \sim 0.29$ (or ellipticity $\epsilon=1-b / a \simeq 0.71$ ). Assuming star counts as a proxy for light, which is reasonable given the spatially uniform, mainly old population of $\mathrm{S} 1$, we estimate a major-axis half-light radius of $R_{e \text {,maj }} \simeq 0.5$, or $\simeq 1.9 \mathrm{kpc}$ at S1's distance (see Section 5). The circularized effective radius is $R_{e, c}=R_{e, \text { maj }} \times \sqrt{b / a} \simeq 0 ! 27$, or $\simeq 1 \mathrm{kpc}$. The fitted elliptical contour at major-axis half-light radius is shown in Figure 6.

\section{Distance}

In this section we use the tip of the RGB to test the hypothesis that S1's distance is compatible with that of
DDO 68; this is a critical step to verify the scenario in which the two systems are physically associated and their observed connection is not a mere chance superposition. Figure 7 shows the CMDs of the DDO 68 portion sampled by our WFC3 data, of S1, selected within the yellow polygon displayed in Figure 6, of a control-field with the same S1's area, and of S1 after subtraction of the field contribution; this was accomplished by "merging" the CMDs of the field and of S1, and by removing from S1 the objects that were closer, in terms of color and magnitude, to the sources detected in the field. We notice that, at magnitudes brighter than F814W $\lesssim 27.5$ (i.e., $\sim 1 \mathrm{mag}$ below the RGB tip), the field contamination to $\mathrm{S} 1$ can be considered negligible. There is a total of 154 stars in the S1 CMD after subtraction of the field contamination.

The red horizontal segment shows the position of the tip of the RGB as determined by applying a Sobel filter to the F814W luminosity function (LF) of stars with $0.6<$ F606W-F814W $<$ 1.2 in DDO 68 (following, e.g., Bellazzini et al. 2011). The RGB tip of DDO 68 is consistent with that visually identified in the S1's CMD, indicating that the two systems are qualitatively at the same distance. The low number of stars populating the RGB of S1 prevents a sensible quantitative determination of the tip position in this system (see, e.g., Madore \& Freedman 1995; Bellazzini et al. 2002, for discussion). Moreover, even in the case of well populated RGBs, the final uncertainty in the distance modulus is of order $\pm 0.1 \mathrm{mag}$ (see below), corresponding to 


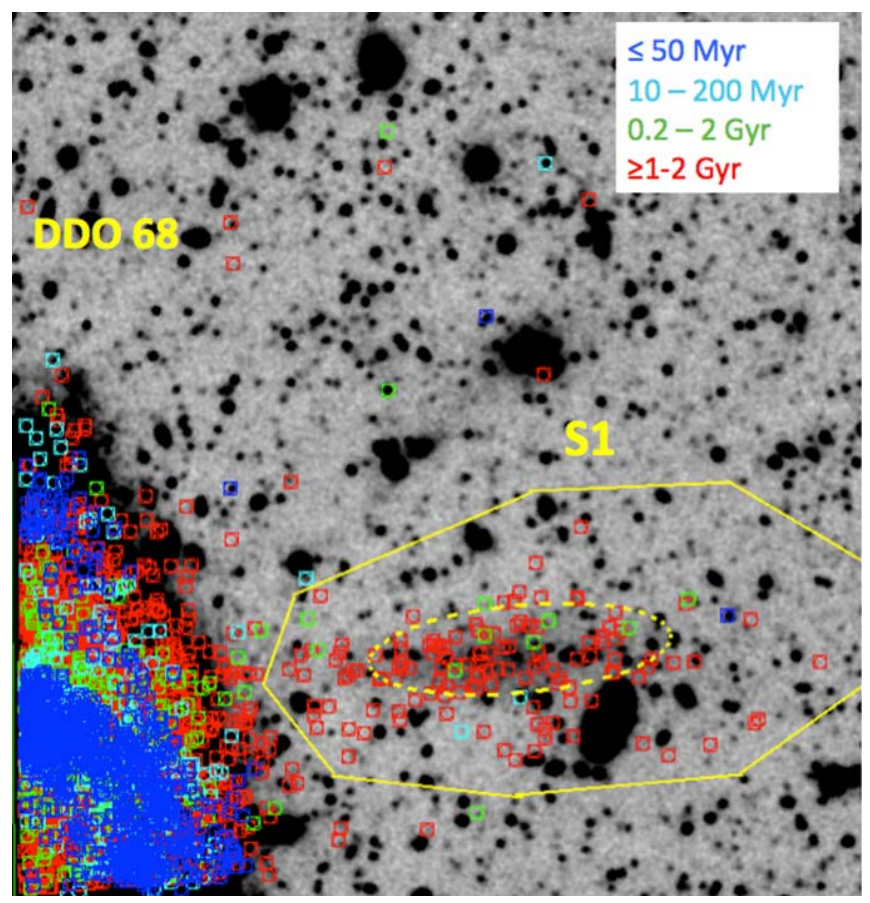

Figure 6. Portion of the LBT/LBC image presented in Annibali et al. (2016) with superimposed stars resolved in the WFC3 data. The displayed image portion has the same field of view as our WFC3 images. Blue, cyan, green, and red denote different age intervals, as selected from the CMD in Figure 5: $\lesssim 50 \mathrm{Myr}$, in the ranges 10-200 Myr and $200 \mathrm{Myr}-2 \mathrm{Gyr}$, and older than 1-2 Gyr. The yellow continuous polygin encloses S1's extension as visually identified from the resolved star map, while the dashed contour is the fitted ellipse at major-axis half-light radius.

$\simeq 0.5 \mathrm{Mpc}$ at the distance of DDO 68 . This would not provide any additional information with respect to the direct comparison shown in Figure 7. We will use synthetic CMDs in Section 6 to provide further support to the agreement between DDO 68's and S1's distances.

To derive DDO 68's distance, we convert the WFC3 F606W, F814W magnitudes into Johnson-Cousins $V, I$ applying the transformations of Harris (2018). We then apply a Sobel filter to the I LF and, after correcting for a foreground extinction of $A_{I}=0.028$ (Schlafly \& Finkbeiner 2011), we obtain $I_{\mathrm{TRGB}, 0}=26.49$. If we input the RGB tip color, $V-I_{\mathrm{TRGB}, 0}=1.04$, into Equation (2) of Bellazzini (2008), we obtain an expected absolute tip magnitude of $M_{I, \mathrm{TRGB}}=$ -4.05 ; this implies a distance modulus of $(m-M)_{0}=30.54 \pm$ 0.12 , corresponding to a distance of $D=12.8 \pm 0.7 \mathrm{Mpc}$. This value is fully consistent within the errors with the distance of $D \sim 12.65 \mathrm{Mpc}$ determined by Sacchi et al. (2016). At this distance, the projected distance between the center of DDO 68 and the center of the stream is $\sim 6 \mathrm{kpc}$.

\section{SFH of S1}

The SFH of S1 was derived through the synthetic CMD method (Tosi et al. 1991) using the Star Formation Evolution Recovery Algorithm (SFERA; see e.g., Cignoni et al. 2015; Sacchi et al. 2018), which is a hybrid-genetic algorithm (genetic plus simulated annealing) based on a Poissonian approach. Synthetic CMDs were constructed starting from the PARSEC-COLIBRI stellar isochrones (Bressan et al. 2012; Marigo et al. 2017), assuming a Kroupa initial mass function
(Kroupa 2001) from 0.1 to $300 M_{\odot}$, a $30 \%$ binary fraction, ${ }^{8}$ and metallicities in the range $Z=0.0001-0.008$ (i.e., from $\sim 1 / 150$ th solar to $\sim$ half solar). In the color-magnitude plane, the isochrones were shifted assuming a distance modulus of $(m-M)_{0}=30.54$ (see Section 5) and a foreground reddening of $E(B-V)=0.016$ (Schlafly \& Finkbeiner 2011), while internal reddening within $\mathrm{S} 1$ is expected to be negligible given the lack of large amounts of gas at its position (Cannon et al. 2014) and the extremely low metallicity of DDO 68. The models were convolved with photometric errors and incompleteness as evaluated from artificial star tests (Section 2.1). Synthetic CMDs were compared on a statistical way to the field-decontaminated CMD of S1 shown in the bottom right panel of Figure 7. The search for the solution was done considering only regions of the CMD above the $50 \%$ completeness limit, translating into a magnitude limit of $\mathrm{F} 814 \mathrm{~W} \sim 28$.

Given the poor statistics affecting the CMD of S1, we adopted in the first place the simplest possible approach and fitted the CMD with simple stellar populations (SSPs) for a wide range of ages (from a few hundred Myr to $13 \mathrm{Gyr}$ ) and metallicities $\left(Z=10^{-4}-8 \times 10^{-3}\right)$. From this analysis, the best agreement is provided by a $7 \pm 1$ Gyr old SSP with metallicity of $Z=10^{-4}$; the resulting stellar mass is $1.6 \pm 0.9 \times 10^{6} M_{\odot}$. The comparison between the observed and the synthetic CMDs is shown in Figure 8. We notice that, despite the good match in the RGB phase, the model fails in accounting for the observed TP-AGB stars at magnitudes just brighter than the RGB tip. Indeed, dwarf galaxies are known to host a quite complex and overly continuous SF, and an SSP fit may not provide an optimal description of the S1 evolution. Therefore, we performed a more sophisticated analysis based on the assumption of a complex SFH, as described in the following.

To simulate a complex $\mathrm{SFH}$, we adopted five age bins ( $\leqslant 100 \mathrm{Myr}, \quad 100-500 \mathrm{Myr}, \quad 500 \mathrm{Myr}-2 \mathrm{Gyr}, \quad 2-6 \mathrm{Gyr}$, and $6-13.7 \mathrm{Gyr}$ ), and let the metallicity freely vary in the range $Z=10^{-4}-8 \times 10^{-3}$, with the only constraint that it could not decrease by more than $25 \%$ with respect to an older adjacent bin. The choice of just five coarse age bins is motivated by the low number of stars in the CMD, the large photometric errors, and the reached magnitude limit of just $\sim 1.5$ mag below the RGB tip. Indeed, from the existence of an RGB we know that stars older than 1-2 Gyr are present in S1, but we are not able to infer in great detail the SFH from the age of the universe to $\sim 2$ Gyr ago.

The best-fit solution from SFERA is presented in Figure 9; in the top panels, we show the empirical and the model CMDs in the form of Hess diagrams, i.e., density plots, and the residuals in units of Poisson uncertainties $(($ data-model $) / \sqrt{\text { model }})$. As visible from the residuals, the model reproduces quite well the observed CMD above F814W $\sim 28$. The TP-AGB star region at $\mathrm{F} 814 \mathrm{~W} \sim 26, \mathrm{~F} 606 \mathrm{~W}-\mathrm{F} 814 \mathrm{~W} \gtrsim 1.2$ is better populated than in the previous SSP-fit case, although a shortage of counts is still present in the synthetic CMD. However, we do not consider this partial discrepancy as a problem here, as it is known that this evolutionary phase is very difficult to model and still lacks proper calibration over the whole range of metallicity regimes.

The middle and bottom panels of Figure 9 show the resulting metallicity and star formation behaviors as a function of look-back

\footnotetext{
8 As shown by Cignoni et al. (2016), the assumed binary fraction does not have a significant impact on the SFH results.
} 

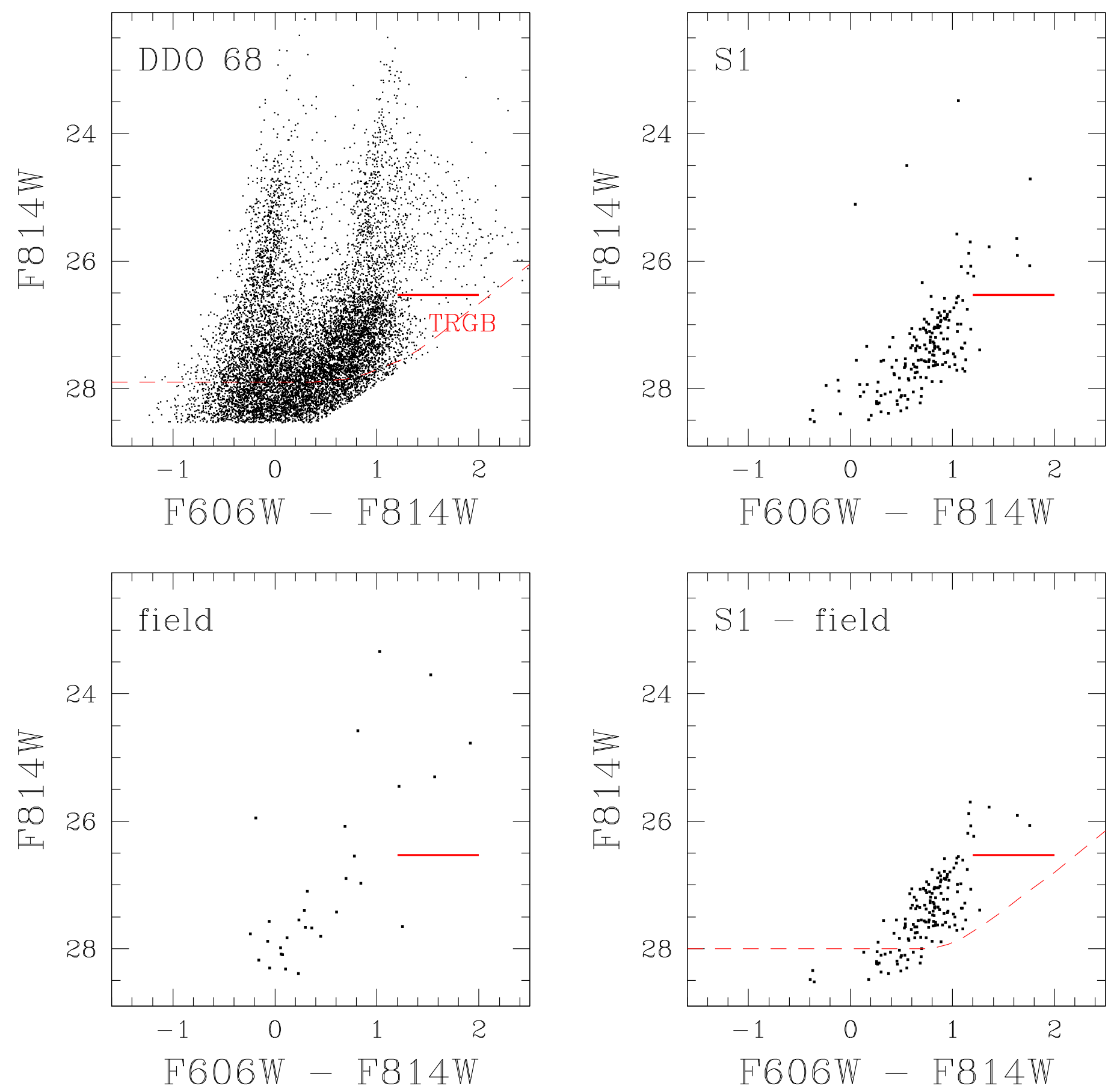

Figure 7. CMDs for the portion of DDO 68 sampled by our WFC3 data (top left panel), for the S1 system, selected to be within the yellow polygon displayed in Figure 6 (top right), for a control-field with the same S1's area (bottom left), and for S1 minus the contribution of the field (bottom right). The dashed curves indicate the $50 \%$ completeness levels for DDO 68 and for S1.

time. The solution recovered by SFERA implies that the vast majority of the SF activity occurred at epochs earlier than $\sim 500 \mathrm{Myr}$ ago; the peak of the activity is predicted to have occurred in the 2-6 Gyr bin, while the adjacent $500 \mathrm{Myr}-2 \mathrm{Gyr}$ and $6-13.7$ Gyr intervals were characterized by a lower SF. As already expected from the CMD appearance in Figure 7, the SF rate in S1 was compatible with zero at epochs more recent than $\sim 0.5 \mathrm{Gyr}$ ago. At earlier epochs we are not able to infer the details of the SFH with the data in our hands; however, the total formed stellar mass of $(1.27 \pm 0.41) \times 10^{6} M_{\odot}$ can be considered a quite robust result and is in reasonable agreement with the estimates from both the SSP-fitting and the integrated light in Annibali et al. (2016).

Concerning metallicity, the best-fit solution implies a continuous increase in $Z$ starting from $\sim 0.0006$ at the oldest look-back times and reaching up to $Z \sim 0.006$ ( $\sim 40 \%$ solar) at 

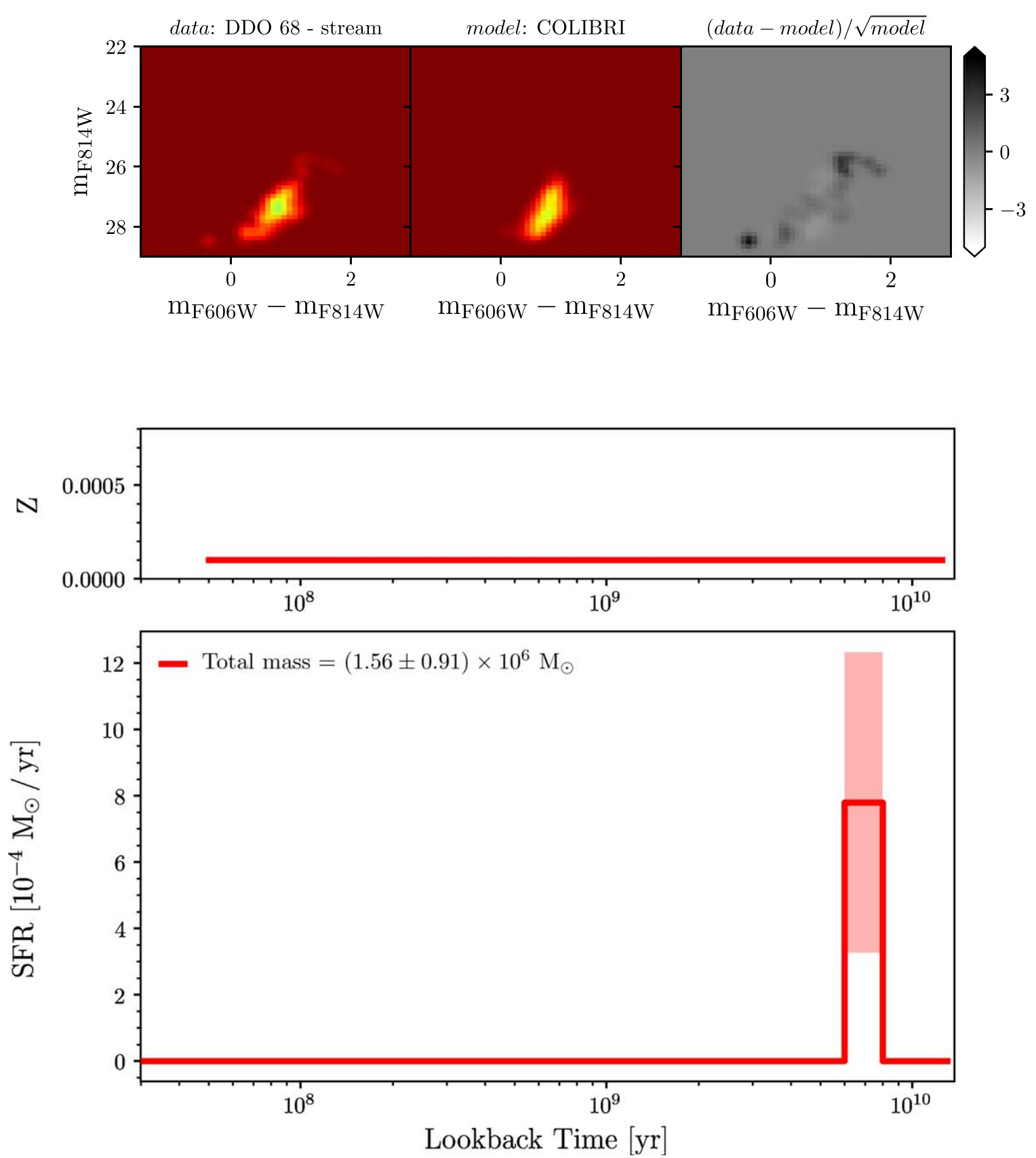

Figure 8. Results from a simple stellar population (SSP) fit analysis applied to S1. The top panels display the Hess diagrams of the observed CMD, of the best-fit SSP and of the residuals. The middle and bottom panels provide the metallicity $\left(Z=10^{-4}\right)$ and age $(7 \pm 1 \mathrm{Gyr})$ of the best-fit SSP. The total formed stellar mass is reported in the bottom panel.

recent epochs. This value is significantly higher than the $Z \sim 0.0004(\sim 2 \%)$ solar metallicity measured in DDO 68's $\mathrm{H}$ II regions, and also much higher than expected for a $\sim 10^{6} M_{\odot}$ stellar mass galaxy. Admittedly, given the strong agemetallicity degeneracy in the RGB phase combined with the relatively large photometric errors of our data, we can not claim a robust constraint on metallicity at epochs earlier than $1 \mathrm{Gyr}$ ago. At more recent epochs, when the rate was compatible with zero, the metallicity is not well constrained either, but is very unlikely to have increased much. Notice that the reddeningmetallicity degeneracy is not expected to play a significant role given the most likely low internal extinction within S1.
In order to test the effect of metallicity on our solution, we performed additional simulations imposing a $Z=0.0004$ metallicity upper limit. The results from this second run are shown in Figure 10 following the same format of Figure 9. The total mass of formed stars is $(1.28 \pm 0.49) \times 10^{6} M_{\odot}$. The new solution still provides a satisfactory agreement between the observed and the synthetic CMDs, with the only difference of a worse match in the TP-AGB star region with respect to the previous run. Indeed, the presence of these stars may have driven SFERA toward the relatively metal-rich solution obtained in the first run. However, that the SFH and the total stellar mass obtained in the two different runs are in agreement 

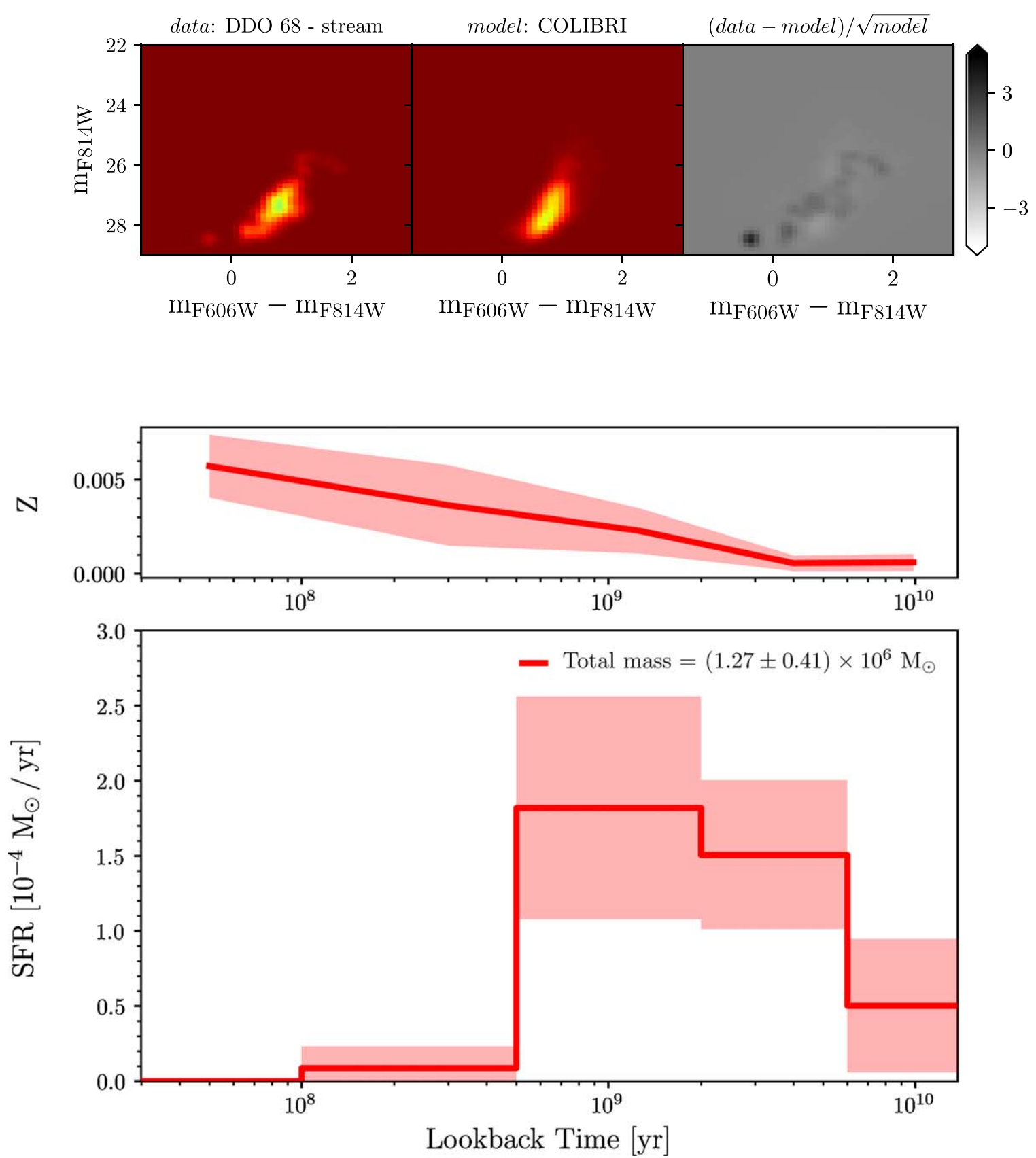

Figure 9. Results of the SFERA procedure for the SFH recovery in S1. Top panels: Hess diagrams of the observed CMD, of the best-fit model, and of the residuals. Middle panel: derived metallicity behavior as a function of look-back time. Bottom panel: derived star formation rate as a function of look-back time. The total formed stellar mass is indicated.

with each other, within the uncertainties, indicates that these results are robust against metallicity uncertainties.

\section{Discussion and Conclusions}

We have presented new HST WFC3 imaging in F606W (V) and $\mathrm{F} 814 \mathrm{~W}(I)$ of a low surface brightness stream-like system, dubbed S1, that is associated with the dwarf galaxy DDO 68 and that was identified for the first time in deep LBT/LBC imaging. Previously acquired HST ACS/WFC images centered on DDO 68 covered a small portion of S1, showing that it was resolved into individual stars, but lacking sufficient statistics to probe its distance and definitively demonstrate its physical association with the dwarf galaxy. The new WFC3 data cover the entire S1 extension and provide a CMD sufficiently populated to assert that $\mathrm{S} 1$ is compatible with DDO 68's distance of $12.8 \pm 0.7 \mathrm{Mpc}$. This result rules out the possibility of a chance superposition and demonstrates that $\mathrm{S} 1$ is indeed physically associated to DDO 68.

While DDO 68 hosts stars of all ages, from $\lesssim 10$ Myr up to several Gyr old stars, the dominant feature of S1 consists of RGB stars, with ages older than $\gtrsim 1-2$ Gyr; a few brighter intermediate-age or old AGB stars are detected, while stars younger than $\sim 200 \mathrm{Myr}$ are not present in the CMD, in agreement with the absence of detected nebular emission (e.g., Pustilnik et al. 2005). The SFH of S1 was derived through synthetic CMDs. The star formation activity at epochs more 

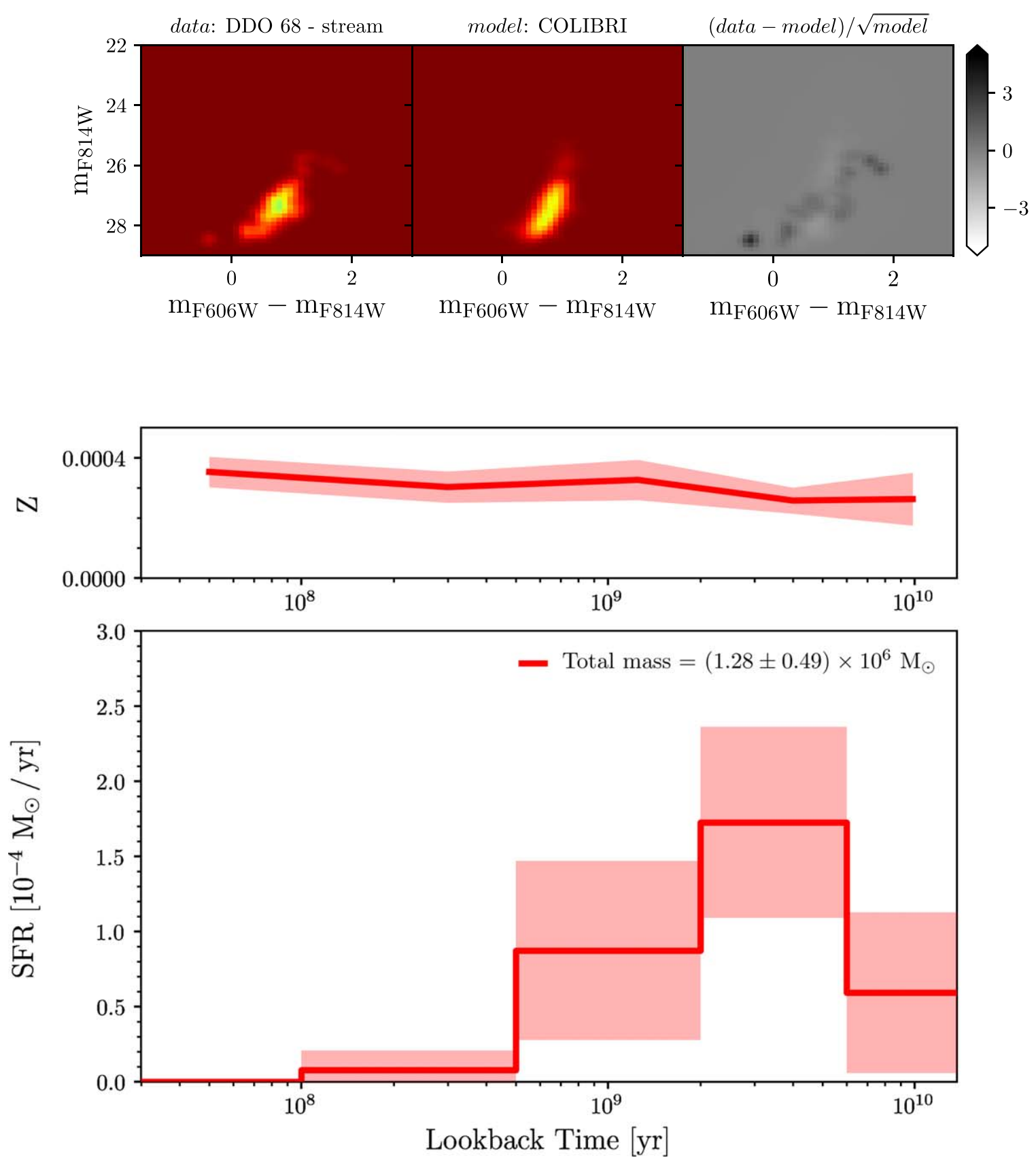

Figure 10. Same as Figure 9, but with the assumption of a $Z=0.0004$ metallicity upper limit.

recent than $\sim 500 \mathrm{Myr}$ is compatible with being null, and the large majority of activity occurred at epochs earlier than $\sim 1$ Gyr ago. While the data in our hands do not allow us to infer the details of the SFH at epochs earlier than $\sim 1$ Gyr ago, the derived total mass of formed stars, $\sim 1.3 \times 10^{6} M_{\odot}$, can be considered a quite robust result.

$\mathrm{S} 1$ is comparable in terms of stellar mass to the two most classic dSph satellites of the Milky Way, Draco, and Ursa Minor (e.g., McConnachie 2012). However S1, with a majoraxis half-light radius of $R_{e \text {, maj }} \simeq 1.9 \mathrm{kpc}$, is significantly more extended than Draco and Ursa Minor, with total major-axis diameters of $\sim 0.2 \mathrm{kpc}$. Indeed, from a comparison with the dwarfs in the compilation of McConnachie (2012), S1 appears exceptionally extended for its luminosity $\left(M_{r}=-9.0 \pm 0.4\right)$, and comparable only to the dSph galaxy And XIX (see Figure 6 of that paper). S1 is also remarkable in terms of its high ellipticity: in the McConnachie compilation, only 4 galaxies out of 110 have $\epsilon \gtrsim 0.7$.

All these properties suggest that $S 1$ has been deeply transformed under the effect of DDO 68's gravitational potential. This scenario is supported by $N$-body simulations only including the collisionless component (stars and dark matter), which are able to reproduce the observed optical properties of S1 (shape, extension, surface brightness) with the recent accretion by DDO 68 of a satellite with total (stellar plus dark matter) mass lower by a factor 150 than the total mass of DDO 68 (Annibali et al. 2016). It is worth noting that the stellar mass ratio between $\mathrm{S} 1$ and DDO 68 is $\approx 1.3 \times 10^{6} /$ $1.3 \times 10^{8}=1 / 100$, which is comparable to the mass ratio between satellite and main galaxy in the simulation. Based on estimates of the stellar-to-halo mass relation for dwarf galaxies (Read et al. 2017), we would expect the ratio between the halo 
masses to be higher than $1 / 150$, but it is possible that the satellite's halo has been largely stripped in the early phases of the interaction with DDO 68.

Existing Very Large Array (VLA) and Green Bank Telescope (GBT) H I data (Cannon et al. 2014) show modest $\mathrm{H} \mathrm{I}$ column densities at the position of $\mathrm{S} 1\left(N_{\mathrm{H} \mathrm{I}} \lesssim 10^{20} \mathrm{~cm}^{-2}\right)$; however, it is not possible to ascertain if any gas is in fact associated with $\mathrm{S} 1$, which turns out to be completely embedded into DDO 68's H I emission due to its close proximity to the dwarf galaxy main body. Admittedly, the lack of stellar populations younger than $\sim 500 \mathrm{Myr}$ would suggest $\mathrm{S} 1$ to be a gas-poor system.

A possible scenario is that the $\mathrm{SF}$ in $\mathrm{S} 1$ was quenched some hundred Myr ago through gas stripping by DDO 68. Nevertheless, it is very unlikely that $\mathrm{S} 1$ had any significant role in keeping the present-day gas metallicity of DDO 68 so low. The total H I gas mass of DDO 68 was estimated to be $\sim 10^{9} M_{\odot}$ by Cannon et al. (2014); even if S1's gas content was a factor of ten more massive than its stellar mass of $\sim 10^{6} M_{\odot}$, its contribution to DDO 68's gas mass would amount to just $1 \%$, too small to significantly dilute the metallicity of DDO 68's interstellar medium. Most likely, the culprit of DDO68's extremely low metallicity is the already merged DDO 68 B system, with a mass of one tenth that of DDO 68's main body.

The case of S1 is particularly relevant in the light of the predictions of the hierarchical galaxy formation process. Darkmatter-only simulations in a $\Lambda \mathrm{CDM}$ cosmology predict that substructures persist within halos down to the resolution limit of the simulations (Diemand et al. 2008); hydrodynamical simulations including different sources of feedback (e.g., Wheeler et al. 2015, 2018) have then attempted to predict the fraction of sub-halos that hosts in fact star formation. For instance, according to Dooley et al. (2017) we expect a $\sim 3 \times 10^{8} M_{\odot}$ stellar mass galaxy (i.e., with mass not too far from that of DDO 68) to host 1-2 satellites with stellar masses above $10^{5} M_{\odot}$. These predictions are in excellent agreement with the case of DDO 68, which is accreting a ten times smaller body (DDO 68 B) and another smaller $10^{6} M_{\odot}$ stellar mass system (S1).

These data are associated with the HST GO program 14716 (PI: F. Annibali). Support for program number 14716 was provided by NASA through a grant from the Space Telescope Science Institute, which is operated by the Association of Universities for Research in Astronomy under NASA contract NAS5-26555. F.A., M.C., and M.T. kindly acknowledge funding from INAF PRIN-SKA-2017 program 1.05.01.88.04. We thank the anonymous referee for her/his useful comments and suggestions.

Facilities: HST(ACS and WFC3), LBT(LBC).

Software: Drizzlepac (Gonzaga et al. 2012), Dolphot (Dolphin 2000).

\section{ORCID iDs}

F. Annibali (1) https://orcid.org/0000-0003-3758-4516

M. Bellazzini (1) https://orcid.org/0000-0001-8200-810X

M. Correnti (i) https://orcid.org/0000-0001-6464-3257
M. Tosi (i) https://orcid.org/0000-0002-0986-4759

M. Cignoni (1) https://orcid.org/0000-0001-6291-6813

A. Aloisi 1 (1) https://orcid.org/0000-0003-4137-882X

D. Calzetti (1) https://orcid.org/0000-0002-5189-8004

L. Ciotti (i) https://orcid.org/0000-0002-5708-5274

F. Cusano (1) https://orcid.org/0000-0003-2910-6565

C. Nipoti (1) https://orcid.org/0000-0003-3121-6616

\section{References}

Amorisco, N. C., Evans, N. W., \& van de Ven, G. 2014, Natur, 507, 335 Annibali, F., La Torre, V., Tosi, M., et al. 2019, MNRAS, 482, 3892

Annibali, F., Nipoti, C., Ciotti, L., et al. 2016, ApJL, 826, L27

Annibali, F., Tosi, M., Aloisi, A., van der Marel, R. P., \& Martinez-Delgado, D. 2012, ApJL, 745, L1

Bellazzini, M. 2008, MmSAI, 79, 440

Bellazzini, M., Beccari, G., Oosterloo, T. A., et al. 2011, A\&A, 527, A58

Bellazzini, M., Ferraro, F. R., Origlia, L., et al. 2002, AJ, 124, 3222

Belokurov, V., Zucker, D. B., Evans, N. W., et al. 2006, ApJL, 642, L137

Bressan, A., Marigo, P., Girardi, L., et al. 2012, MNRAS, 427, 127

Calzetti, D., Lee, J. C., Sabbi, E., et al. 2015, AJ, 149, 51

Cannon, J. M., Johnson, M., McQuinn, K. B. W., et al. 2014, ApJL, 787, L1

Cicuéndez, L., \& Battaglia, G. 2018, MNRAS, 480, 251

Cignoni, M., Sabbi, E., van der Marel, R. P., et al. 2015, ApJ, 811, 76

Cignoni, M., Sabbi, E., van der Marel, R. P., et al. 2016, ApJ, 833, 154

Crnojević, D., Sand, D. J., Bennet, P., et al. 2019, ApJ, 872, 80

Crnojević, D., Sand, D. J., Spekkens, K., et al. 2016, ApJ, 823, 19

Deustua, S. E., \& Mack, J. 2018, Instrument Science Rep., ACS 2017-10

Diemand, J., Kuhlen, M., Madau, P., et al. 2008, Natur, 454, 735

Dolphin, E. A. 2000, PASP, 112, 1383

Dooley, G. A., Peter, A. H. G., Yang, T., et al. 2017, MNRAS, 471, 4894

Ekta, Chengalur, J. N., \& Pustilnik, S. A. 2008, MNRAS, 391, 881

Gonzaga, S., Hack, W., Fruchter, A. S., \& Mack, J. (ed.) 2012, The DrizzlePac Handbook (Baltimore, MD: STScI)

Harris, W. E. 2018, AJ, 156, 296

Ibata, R., Irwin, M., Lewis, G., Ferguson, A. M. N., \& Tanvir, N. 2001, Natur, 412, 49

Izotov, Y. I., \& Thuan, T. X. 2009, ApJ, 690, 1797

Kacharov, N., Battaglia, G., Rejkuba, M., et al. 2017, MNRAS, 466, 2006

Koposov, S. E., Walker, M. G., Belokurov, V., et al. 2018, MNRAS, 479, 5343 Kroupa, P. 2001, MNRAS, 322, 231

Lee, J. C., Salzer, J. J., \& Melbourne, J. 2004, ApJ, 616, 752

Madore, B. F., \& Freedman, W. L. 1995, AJ, 109, 1645

Marigo, P., Girardi, L., Bressan, A., et al. 2017, ApJ, 835, 77

Martínez-Delgado, D., Gabany, R. J., Crawford, K., et al. 2010, AJ, 140, 962 Martínez-Delgado, D., Romanowsky, A. J., Gabany, R. J., et al. 2012, ApJL, 748, L24

McConnachie, A. W. 2012, AJ, 144, 4

McConnachie, A. W., Irwin, M. J., Ibata, R. A., et al. 2009, Natur, 461, 66 Moiseev, A. V. 2014, AstBu, 69, 1

Pustilnik, S. A., Kniazev, A. Y., \& Pramskij, A. G. 2005, A\&A, 443, 91

Pustilnik, S. A., Makarova, L. N., Perepelitsyna, Y. A., Moiseev, A. V., \& Makarov, D. I. 2017, MNRAS, 465, 4985

Pustilnik, S. A., \& Tepliakova, A. L. 2011, MNRAS, 415, 1188

Read, J. I., Iorio, G., Agertz, O., \& Fraternali, F. 2017, MNRAS, 467, 2019

Rich, R. M., Collins, M. L. M., Black, C. M., et al. 2012, Natur, 482, 192

Ryan, R. E., Jr., Deustua, S., \& Sosey, M. 2016, Space Telescope WFC3 Instrument Science Rep., 2016-001

Sacchi, E., Annibali, F., Cignoni, M., et al. 2016, ApJ, 830, 3

Sacchi, E., Cignoni, M., Aloisi, A., et al. 2018, ApJ, 857, 63

Sand, D. J., Spekkens, K., Crnojević, D., et al. 2015, ApJL, 812, L13

Schlafly, E. F., \& Finkbeiner, D. P. 2011, ApJ, 737, 103

Stierwalt, S., Liss, S. E., Johnson, K. E., et al. 2017, NatAs, 1, 25

Tikhonov, N. A., Galazutdinova, O. A., \& Lebedev, V. S. 2014, AstL, 40, 1

Tosi, M., Greggio, L., Marconi, G., \& Focardi, P. 1991, AJ, 102, 951

Wheeler, C., Hopkins, P. F., Pace, A. B., et al. 2018, arXiv:1812.02749

Wheeler, C., Oñorbe, J., Bullock, J. S., et al. 2015, MNRAS, 453, 1305

Williams, B. F., Lang, D., Dalcanton, J. J., et al. 2014, ApJS, 215, 9 